\title{
A Candida albicans chaperonin subunit (CaCct8p) as a suppressor of morphogenesis and Ras phenotypes in C. albicans and Saccharomyces cerevisiae
}

\author{
Felicitas Rademacher, Verena Kehren, Volker R. Stoldt \\ and Joachim F. Ernst
}

\begin{abstract}
Author for correspondence: Joachim F. Ernst. Tel./Fax: +49 2118115176.
\end{abstract}
e-mail: joachim.ernst@uni-duesseldorf.de

Institut für Mikrobiologie and Biologisch-

Medizinisches

Forschungszentrum,

Heinrich-Heine-Universität, Universitätsstr. 1/26.12,

D-40225 Düsseldorf,

Germany

\begin{abstract}
Saccharomyces cerevisiae and the pathogen Candida albicans can be induced to undergo morphogenesis from a yeast to a filamentous form. A C. albicans gene (CaCCT8) was identified encoding a subunit of the Cct chaperonin complex, whose expression prevents filament formation in both fungi without interfering with growth of the yeast form. In S. cerevisiae, pseudohyphal growth induced by Ras2 ${ }^{\mathrm{Va}}{ }^{19}$, by overproduction of Phd1p or by expression of the C. albicans EFG1 gene, was blocked by CaCct8p and its $\mathbf{N}$-terminally deleted derivative CaCct8- $\triangle 1 \mathrm{p}$; in contrast, pseudohyphal induction by other components (Cph1p, Cdc42p) could not be suppressed, indicating that morphogenesis per se is not inhibited. CaCCT8 expression also interfered with other Ras $2 p^{\text {val19 }}$ phenotypes, including heat sensitivity, lack of glycogen accumulation and lack of sporulation. In C. albicans, overproduction of CaCct8p effectively blocked hyphal morphogenesis induced by starvation conditions and by serum. The results suggest that the activity of a component in the Ras2p signal transduction pathway is suppressed by excess chaperonin subunits. This component may be a novel folding target for the Cct complex. In agreement with this hypothesis, disruption of one of the two CaCCT8 alleles in C. albicans led to defective hyphal morphogenesis.
\end{abstract}

Keywords: Candida, morphogenesis, dimorphism, chaperonins, Cct complex

\section{INTRODUCTION}

An ability to interconvert between different growth forms is considered an important virulence trait of many pathogenic fungi. The human pathogen Candida albicans is able to grow as a budding yeast, but depending on environmental conditions, growth can also occur in a filamentous form. At high temperatures and induced by a variety of compounds, such as serum components, $C$. albicans forms true unconstricted hyphae consisting of mononucleate cells separated by septae (reviewed by Odds, 1988). The molecular mechanisms leading to the establishment of yeast and hyphal growth forms are

Abbreviations: MAP, mitogen-activated protein; PKA, protein kinase A. The GenBank accession number for the sequence reported in this paper is U37371. poorly understood. An evolutionarily conserved mitogen-activated protein (MAP) kinase cascade that is essential for filamentous growth of the yeast Saccharomyces cerevisiae is not absolutely required for morphogenesis and virulence of C. albicans. Mutants lacking the MAP kinase components Cst20p, Hst7p and Cph1p show normal hyphal development in liquid media and during serum induction, but reduced hyphal formation on certain solid media; $h s t 7$ mutants are fully virulent in the mouse infection model, while cst 20 and $c p h 1$ mutants only show an attenuated virulence (Köhler \& Fink, 1996; Leberer et al., 1996; Liu et al., 1994; Lo et al., 1997). Recently, Efg1p, an essential regulator of $C$. albicans morphogenesis and virulence, has been identified and belongs to a novel class of bHLH proteins that control morphogenetic processes in fungi (Lo et al., 1997; Stoldt et al., 1997).

Although $S$. cerevisiae does not form true septated 
hyphae, some diploid strains are able to bud in a unipolar fashion with elongated cells that remain attached, thereby leading to a hypha-like appearance (pseudohypha) (Gimeno et al., 1992). The MAP kinase module containing Ste20p, Ste11p, Ste7p and Kss1p, which activates the transcription factor Ste12p, is required for pseudohyphal morphogenesis (Liu et al., 1993; Madhani et al., 1997). The Tec1 transcription factor acts in concert with Ste12p to regulate genes involved in filamentous growth (Gavrias et al., 1996; Madhani \& Fink, 1997). Activated Ras2 protein stimulates morphogenesis via Cdc42p, Ste20p and the MAP kinase pathway, possibly in a PKA (protein kinase A)independent manner (Mösch et al., 1996). In contrast, recent evidence strongly suggests that a cAMP-dependent pathway is crucial for pseudohyphal formation. Mutations in the MAP kinase pathway can be suppressed by hyperactivation of the cAMP pathway, e.g. by alleles of GPA2 encoding a constitutively active Gprotein $\alpha$-subunit or by externally added cAMP (Kübler et al., 1997; Lorenz \& Heitman, 1997). Similarly, overproduction of Phd1p, a homologue of the C. albicans Efg1 protein, is able to suppress mutations in the MAP kinase pathway (Lo et al., 1997).

Filamentous growth in fungi is the consequence of a polarized assembly of components in cells, requiring cytoskeletal proteins, including actin and tubulins (reviewed by Kron \& Gow, 1995). It has been shown that in eukaryotes the cytosolic chaperonin complex containing Tcp-1 (Cct1p) has a narrow substrate specificity and is known to fold actin and tubulin monomers (reviewed by Kubota et al., 1995). Cct is a heterooligomer complex consisting of eight different $\mathrm{Cct}$ subunits, which in yeast are encoded by essential genes (reviewed by Stoldt et al., 1996). Tcp-1 (Cct1p) overproduction is known to partially suppress actin mutations (Ursic et al., 1994) and recently, CCT6 (TCP20) overexpression was shown to suppress mutations in TOR2 affecting the polarized distribution of actin (Schmidt et al., 1996). Cct can bind newly synthesized actin and tubulin and a restricted set of minor unidentified polypeptides in vivo (Sternlicht et al., 1993). Here we present evidence suggesting that a component of the Ras pathway may be one of the unidentified targets for the Cct complex. We show that an extraneous Cct subunit $(\mathrm{CaCct} 8 \mathrm{p})$, while not affecting yeast growth, suppresses filamentous morphogenesis in C. albicans and $S$. cerevisiae, as well as several Ras phenotypes. Thus, excess chaperonin subunits appear to act as dominant-negative suppressors of the cAMP branch of signal transduction pathways leading to fungal morphogenesis.

\section{METHODS}

Strains and growth conditions. C. albicans strain CAI4 (Fonzi \& Irwin, 1993) was used as host for recombinant plasmids and gene disruption. S. cerevisiae strain MB331-17A, previously referred to as B76 (Stoldt et al., 1997), had been obtained from a cross of MB211-3B ( $\Sigma 1278 \mathrm{~b}$ background) and DBY785 (S288C background) (Brandriss, 1983); the isogenic diploid of
MB331-17A, designated B76/4, developed pseudohyphae in low nitrogen conditions (Stoldt et al., 1997). S. cerevisiae strain L5976 (MATa/MATa ura3-52/ura3-52 leu2::hisG/ leu $2:$ : his $G \operatorname{trp} 1:$ : his $G / \operatorname{trp} 1::$ his $G)$, which is congenic to the $\Sigma 1278$ b background (Gimeno et al., 1992), was provided by H.-U. Mösch (Universität Göttingen, Germany). S. cerevisiae strain B76leu2:: URA3 was constructed by transformation of B76 with a leu2::URA3 disruption fragment, followed by diploidization using a $\mathrm{HO}$-containing plasmid, as described by Stoldt et al. (1997). Fungal transformations were carried out by the lithium acetate method or, for C. albicans, by the spheroplast method (Sherman et al., 1986). Yeast cells were grown in YPD, YP (YPD without glucose), YPGal (YP containing $2 \%$ galactose) or on supplemented SD minimal medium (Sherman et al., 1986). Pseudohyphal growth of $S$. cerevisiae cells was induced on SLAHD plates (Gimeno et al., 1992) containing $50 \mu \mathrm{M}$ ammonium sulfate and amino acids to allow growth of auxotrophic strains, or on SLAHGal plates containing $2 \%$ galactose and $0.1 \%$ glucose to induce expression by the $G A L 1 / 10$ promoter. Pseudohyphal growth was inspected microscopically after $2 \mathrm{~d}$ at $30^{\circ} \mathrm{C}$ using a Zeiss Axioscop. To induce hyphae, C. albicans cells were pregrown at $30^{\circ} \mathrm{C}$ in YPD or in $1 \%$ Casamino acids (for pBI1-CCT8) to exponential growth phase, starved for $1 \mathrm{~h}$ at $30^{\circ} \mathrm{C}$ in $0.45 \%$ $\mathrm{NaCl} / 0.335 \%$ yeast nitrogen base without amino acids $(\mathrm{pH} 5 \cdot 5) / 10 \mathrm{mM}$ proline and then diluted $\left(\mathrm{OD}_{600} 0 \cdot 1\right)$ into the same medium containing $1 \mathrm{mM} \mathrm{N}$-acetylglucosamine at $\mathrm{pH} 6.5$ and $37^{\circ} \mathrm{C}$. Alternatively, cells were diluted into YP medium containing $5 \%$ horse serum at $37^{\circ} \mathrm{C}$ (Delbrück \& Ernst, 1993). Hyphal formation on solid media was allowed on 'Spider' medium (Liu et al., 1994) or on $2 \%$ agar containing $1 \%$ horse serum, at $37^{\circ} \mathrm{C}$. The phenotypes generated by the dominant $R A S 2^{\text {val19 }}$ mutation were examined essentially as described by Toda et al. (1985), except that during the tests to determine heat sensitivity or glycogen accumulation, transformants were grown in YPD or in YPGal, which induces the GAL1/10 promoter of GAL-CCT fusion genes. Because of poor sporulation of B76/4 transformants, strain VW1D (MATa/MAT $\alpha$ trp1/trp1 ura3-52/ura3-52 leu2-3,112/leu23,112 his $3 /$ his 3 ; obtained from M. Ciriacy, Universität Düsseldorf, Germany) was used as a host to test the effect of CCT genes on sporulation. Sporulation was allowed to proceed in liquid $1 \%$ potassium acetate for $5-7 \mathrm{~d}$.

CCT alleles and expression vectors. $S$. cerevisiae $\mathrm{B} 76 / 4$ was transformed with plasmid p606/1 encoding the $C$. albicans Phd1p homologue Efg1p (Stoldt et al., 1997). The transformant, which showed enhanced pseudohyphal growth on SLAHD plates, was retransformed with a genomic bank of $C$. albicans ATCC 10231 in plasmid pLG89-B1 (Losberger \& Ernst, 1989; Leuker \& Ernst, 1994). From one transformant, in which morphogenesis was suppressed, plasmid pU1, containing a $2.95 \mathrm{~kb}$ Sau3A genomic fragment, was isolated. A shortened $2.8 \mathrm{~kb}$ EcoRI subfragment, in pRS426 (Christianson et al., 1992) (pRS426U1), was shown to be sufficient for suppression; its sequence has the potential to encode a deleted $\mathrm{CaCct} 8 \mathrm{p}$ protein (Cacct8- $\Delta 1$ ) commencing with methionine residue 96 (Stoldt et al., 1996) (5' GAATTCGAAATG; EcoRI site underlined, Met codon in bold). The missing $5^{\prime}$ end of CaCCT 8 was isolated from a genomic bank of C. albicans ATCC 10231 (Losberger \& Ernst, 1989) and sequenced. A BamHI site was constructed upstream of the reconstituted CaCCT8 coding region by PCR mutagenesis (primer PRFGges: 5' TTAGGATCCAATGTCGTTGAAG; BamHI site underlined, start codon in bold); this generated a $3 \mathrm{~kb}$ BamHI fragment carrying CaCCT8.

$C C T$ expression plasmids include insertions of the $2 \cdot 8 \mathrm{~kb}$ 
EcoRI fragment of pU1 (Cacct8- $\Delta 1$ ) into the EcoRI sites of plasmids pRS426 or pRS424 (Christianson et al., 1992) (pRS426U1 or pRS424U1) or YCplac33 (Gietz \& Sugino, 1988) (YCplac33-RFG1); a fortuitous plasmid promoter drives transcription in these plasmids. The $2.8 \mathrm{~kb}$ EcoRI fragment was also placed into the EcoRI site downstream of the GAL10 promoter (Johnston \& Davis, 1984); basic vectors YEplac195 (Gietz \& Sugino, 1988) and pRS424 contained this fusion as Bam HI-EcoRI inserts, resulting in vectors YEplac195Gal10RFG1 and pRS424-Gal10-RFG1, respectively. YEplacGal1/10 contains only the GAL1/10 promoter inserted in YEplac195. Full-length $\mathrm{CaCCT8}$, as a $3.1 \mathrm{~kb}$ BamHI fragment, was inserted into the BamHI site downstream of the GAL1 promoter in YEplac195 (YEplacGal1CCT8) or pRS424 (pRS424-Gal1-CCT8).

A C. albicans expression plasmid carrying $\mathrm{CaCCT8}$ under transcriptional control of the ACT1 promoter, pCARS-ActRFG1, was constructed by joining the $C$. albicans ACT1 promoter as a $1 \mathrm{~kb}$ BamHI-EcoRI fragment (Delbrück \& Ernst, 1993) to the $2.8 \mathrm{~kb}$ EcoRI-BamHI fragment of plasmid pRS426U1 and inserting the fusion into the BamHI site of plasmid pCARS (Kelly et al., 1988). pBI1-CCT8, in which $\mathrm{CaCCT8}$ is regulated by the CaPCK1 promoter, was constructed by first inserting the promoter, as a $1.4 \mathrm{~kb} \mathrm{BamHI}$ Stul fragment of plasmid pCA01 (Leuker et al., 1997) between the BamHI/SmaI sites of pRC2312 (Cannon et al., 1992) to generate $\mathrm{pBI}-1$; the $3.1 \mathrm{~kb}$ Bam HI fragment of YEplacGal1 CCT8 was then transferred into the BglII site of $\mathrm{pBI}-1$ to generate $\mathrm{pBI} 1-\mathrm{CCT} 8$.

Other plasmids. Plasmids pHY2 (STE12-CPH1) (Singh et al., 1994) and pCG38 (PHD1) (Gimeno \& Fink, 1994) have been described. YCplac22RAS2 ${ }^{\text {val19 }}$ was constructed by inserting the $2.9 \mathrm{~kb}$ EcoRI-HindIII fragment of YCpR2V (Gimeno et al., 1992) into YCplac22. pILC5-C, which contains the TEC1 gene on a $U R A 3$ and $2 \mu$ marked plasmid, was obtained from E. Dubois (Université Libre de Bruxelles, Belgium; Laloux $e t$ al., 1994). Plasmid pGAL1-cdc42 ${ }^{\mathrm{val12}}$ carrying the dominant $C D C 42^{\text {val12 }}$ allele (Ziman et al., 1991) was a gift from D. Johnson (University of Vermont, USA). Plasmid pFG(TYA):: lacZ-URA encoding the $F G(T y A):: l a c Z$ reporter fusion (Mösch et al., 1996) was kindly supplied by H.-U. Mösch.

Gene disruption. To place a URA3-containing disruption cassette into CaCCT8, PCR was performed using YEplacGal1CCT8 as template and two primers ( $5^{\prime}$ TTAAGATCTGAAATCAAGCATTTC and 5' TTAAGATCTCATTGGTTTCAACAC; BglII sites underlined) designed to amplify CaCCT8 termini encoding residues 1-264 and the $3^{\prime}$ untranslated region along with plasmid sequences. The PCR product was cut with $B g l I I$ and ligated; into the $B g l I I$ site of the resulting vector we inserted a $3.5 \mathrm{~kb} \mathrm{BamHI}$ fragment of plasmid pCAC (obtained from P. Magee, University of Minnesota, USA), which contains the CaURA3 gene flanked by direct repeats of the Escherichia coli cat gene. From the resulting plasmid, pBLAST-CCT8, the $4.5 \mathrm{~kb}$ Sall fragment carrying the disrupted $\mathrm{CaCCT} 8$ was excised and used to transform C. albicans CAI4 to prototrophy. DNA of transformants was cut with EcoRI and probed, in a Southern blot, with a $0.5 \mathrm{~kb}$ Sall fragment of pRS424U1 containing the $3^{\prime}$ untranslated region of $\mathrm{CaCCT8}$. The wild-type strain yielded the expected fragment of $2.8 \mathrm{~kb}$, while the heterozygous disruptants (FR1-1, 2, 3) contained the $2 \cdot 8 \mathrm{~kb}$ fragment and a $1.9 \mathrm{~kb}$ fragment of the disrupted allele.

\section{RESULTS}

\section{CaCCT8 suppresses pseudohyphal morphogenesis in S. cerevisiae}

We recently identified a C. albicans gene, EFG1, encoding a close homologue of Phd1p, which enhances $S$. cerevisiae pseudohyphal morphogenesis (Stoldt et al., 1997). To identify suppressors of morphogenesis, we retransformed an $S$. cerevisiae transformant expressing EFG1 with a C. albicans genomic library and looked for transformant colonies that did not show pseudohyphal induction. From one of these transformants we recovered a library plasmid (pU1) and analysed its genomic insert by sequencing. The insert was found to contain a deleted version of the C. albicans CaCCT8 gene (Stoldt et al., 1996) encoding a subunit of the cytosolic chaperonin complex on a $2.95 \mathrm{~kb}$ Sau3A fragment (see Methods). The deleted version of $C a C C T 8$, designated Cacct8- $\Delta 1$, has the potential to encode a CaCct8 protein commencing at residue 96 ; a fortuitous promoter located within plasmid sequences appeared to drive transcription of the truncated allele.

The missing $5^{\prime}$ end of the $C a C C T 8$ gene was identified in a partial genomic C. albicans bank using the Cacct8- $\Delta 1$ allele as probe. The $5^{\prime}$ fragment thus isolated was used to reconstitute the full-length $\mathrm{CaCCT8}$ sequence. To allow conditional expression of $\mathrm{CaCCT8}$, a convenient BamHI site was placed immediately upstream of the ATG translational start sequence. This $\mathrm{CaCCT8}$ allele was placed under transcriptional control of the $S$. cerevisiae GAL1 promoter (Johnston \& Davis, 1984).

Transformants of $S$. cerevisiae strain B76/4 (Stoldt et al., 1997) expressing $\mathrm{CaCCT} 8$ on a replicating vector grew in the yeast form at identical rates and with identical cytological appearance compared to control strains carrying an empty vector in all conditions. This result demonstrated that $\mathrm{CaCCT8}$ expression in $S$. cerevisiae did not affect growth in general. In contrast, pseudohyphal morphogenesis induced by EFG1-carrying plasmids was completely blocked by the simultaneous presence of plasmids containing $\mathrm{CaCCT} 8$ or its deleted derivative Cacct8- $\Delta 1$ (Fig. 1). Effective suppression of morphogenesis was obtained with Cacct8- $\Delta 1$ contained in either high-copy-number vectors (pRS426U1, YEplac195Gal10-RFG1) or in a low-copy-number centromeric vector (YCplac33-RFG1).

These results raised the question whether CaCCT8 alleles mediated specific suppression of morphogenesis induced by the C. albicans EFG1 gene or affected morphogenesis in general. Therefore, we tested if pseudohyphal growth induced by a variety of genes, including genes encoding Phd1p (Gimeno \& Fink, 1994), Ras2p $^{\text {val19 }}$ (Gimeno et al., 1992), Cph1p (Singh et al., 1994), Cdc42p (Mösch et al., 1996) and Tec1p (Gavrias et al., 1996), could be suppressed. Morphogenesis induced by the EFG1 homologue PHD1 was effectively blocked by $C a C C T 8$ or Cacct8- $\Delta 1$ (Fig. 1). Similarly, the strong pseudohyphal induction by $R A S 2^{\mathrm{Val19}}$ was effec- 


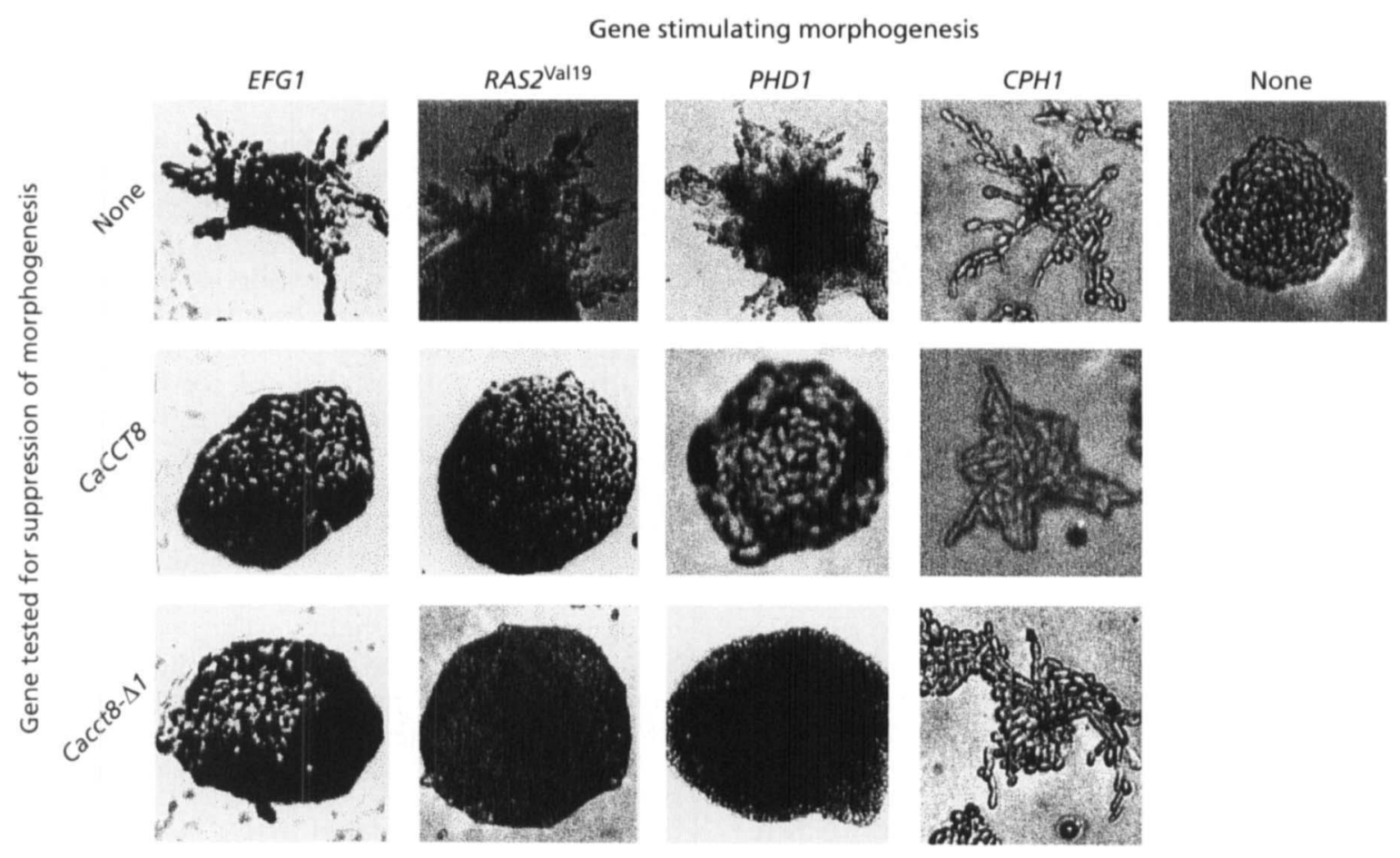

Fig. 1. Colony phenotypes of $S$. cerevisiae transformants. Transformants contained two types of plasmid, one inducing pseudohyphal growth and a second that was tested for suppression of morphogenesis. Colonies of transformants were grown for 2-3 d on SLAHD medium or (if required for induction of the GAL promoter) in SLAHGal low-nitrogen medium. The following combinations of genes (plasmids) were tested, using B76/4 as the host strain: EFG1 (p606/1) and RAS2val19 (YCplac22RAS2 ${ }^{\text {Val19) }}$ in combination with control vector YEplac195, or with CaCCT8 (YEplacGal1CCT8), or with Cacct8- 11 (YCplac33-RFG1); PHD1 (pCG38) and a STE12-CPH1 fusion (pHY2) in combination with control vector YCplac22, or with CaCCT8 (pRS424-Gal1-CCT8), or with Cacct8- $\Delta 1$ (pRS424-Gal10-RFG1). In addition a B76/4 transformant containing two control vectors (YEplac195 and YCplac22) was tested.

\section{Table 1. Regulation of $F G(T y A):: l a c Z$ expression}

Transformants of both strains carried $\mathrm{pFG}(\mathrm{TY} A)$ :: lacZ-URA encoding the $F G(T y A):$ :lacZ reporter fusion, as well as a plasmid containing the indicated gene. Strains were grown on solid SD (high nitrogen) or SLAHD medium containing $50 \mu \mathrm{M} \mathrm{NH} \mathrm{SO}_{4}$ (low nitrogen) and $\beta$-galactosidase levels in cellular extracts were determined as described by Mösch et al. (1996). The activity of at least two transformants, each tested in duplicate, was determined. Activities are expressed relative to the mean value of the control strain B76/4(pRS424) \pm SD.

\begin{tabular}{|c|c|c|c|c|}
\hline \multirow[t]{2}{*}{ Strain } & \multicolumn{2}{|c|}{ Added gene (plasmid) } & \multicolumn{2}{|c|}{ Relative $\beta$-galactosidase activity } \\
\hline & & & High nitrogen & Low nitrogen \\
\hline \multirow[t]{4}{*}{$\mathrm{B} 76 / 4$} & - & (pRS424) & $1.00 \pm 0.19$ & $3.53 \pm 1.86$ \\
\hline & $\operatorname{Cacct} 8-\Delta 1$ & (pRS424U1) & $0.99 \pm 0.53$ & $2 \cdot 79 \pm 1 \cdot 1$ \\
\hline & - & (YEplac112) & $0.56 \pm 0.06$ & $2.88 \pm 0.71$ \\
\hline & $R A S 2^{\mathrm{val19}}$ & $\left(Y_{\text {Cplac22RAS2 }}{ }^{\text {val19 }}\right)$ & $0.83 \pm 0.35$ & $14 \cdot 33 \pm 6 \cdot 93$ \\
\hline \multirow[t]{2}{*}{ L5976 } & - & (pRS424) & $1 \cdot 02 \pm 0 \cdot 14$ & $6.90 \pm 1 \cdot 0$ \\
\hline & Cacct8- $\Delta 1$ & (pRS424U1) & $0.90 \pm 0.42$ & $6.17 \pm 0.75$ \\
\hline
\end{tabular}

tively inhibited (Fig. 1), indicating that suppression by CaCCT8 alleles was not specific for the Efg1p inducer. Suppression of morphogenesis by CaCCT8 alleles was also not specific for the host strain used (B76/4) because it was also observed in the $\Sigma 1298$ b derivative L5976 (data not shown). 
Ras $2 p$ is known to trigger filamentous growth either via Cdc42p and an MAP kinase cascade activating transcription factor Ste12p or via a signalling pathway mediated by cAMP (Kübler et al., 1997; Lorenz \& Heitman, 1997). The C. albicans CPH1 gene encodes a homologue of transcription factor Ste12p which is functional and is activated by the MAP kinase cascade in S. cerevisiae (Singh et al., 1994). Transformants expressing an STE12-CPH1 fusion showed vigorous pseudohyphal growth, which could not be suppressed by $C a C C T 8$ or Cacct8- $\Delta 1$ (Fig. 1). Similarly, transformants of strain B76leu2:: URA3 expressing the dominant active CDC42 $2^{\text {val12 }}$ allele (Ziman et al., 1991; on plasmid pGAL1-cdc42 ${ }^{\text {val12 }}$ ) showed enhanced morphogenesis (Mösch et al., 1996), but this phenotype could not be suppressed by simultaneous expression of Cacct8- $\Delta 1$ (on plasmid pRS424-Gal10-RFG1; data not shown). TEC1 encodes a transcription factor acting in concert with Ste12p to regulate genes required for pseudohyphal growth (Gavrias et al., 1996; Madhani \& Fink, 1997). Overexpression of TEC1 (on plasmid pILC5-C) in strain B76/4 stimulated pseudohyphal development as expected, but morphogenesis could not be suppressed by simultaneous expression of Cacct8- $\Delta 1$ (on plasmid pRS424-Gal10-RFG1; data not shown).

These results indicated that CaCCT8 alleles did not affect morphogenesis in general and suggested that the putative target of suppression was not a part of the MAP kinase pathway, but was possibly located in the cAMP pathway stimulated by Ras $2 p$. We confirmed that the suppression of morphogenesis was not due to a block of the MAP kinase cascade by examining the activity of the $F G(T y A)$ promoter, which depends on this pathway (Lorenz \& Heitman, 1997; Mösch et al., 1996). The expression of the $F G(T y A):: \operatorname{lac} Z$ construct was increased four- to sevenfold in strains B76/4 and L5976 by growth in low nitrogen conditions, as expected, but the simultaneous expression of Cacct8- $\Delta 1$ did not significantly influence expression levels (Table 1).

\section{CaCCT8 alleles suppress morphogenesis-unrelated phenotypes of Ras $2 p^{\text {val19 }}$}

Activation of the Ras pathway in S. cerevisiae not only stimulates pseudohyphal morphogenesis, but also leads to heat sensitivity, lack of glycogen/trehalose accumulation and lack of sporulation (Toda et al., 1985). To explore if these additional phenotypes were also affected by $\mathrm{CaCCT} 8$ expression, we used double transformants of strain $\mathrm{B} 76 / 4$ carrying a $R A S 2^{\mathrm{Val19}}$-containing plasmid as well as a plasmid expressing the CaCCT8 or Cacct8$\Delta 1$ alleles. As controls, transformants carrying an empty

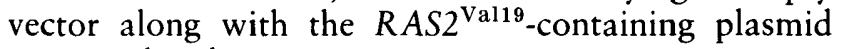
were analysed.

We tested heat sensitivity of transformants by heat shock of stationary cells at $50^{\circ} \mathrm{C}$, followed by plating of cells on SD minimal medium. As expected, the presence of the RAS2 $2^{\text {val19 }}$ allele rendered transformants heatsensitive. This sensitivity was completely reversed by coexpression of $\mathrm{CaCCT} 8$ and Cacct8- $\Delta 1$ (Fig. 2a). (a)
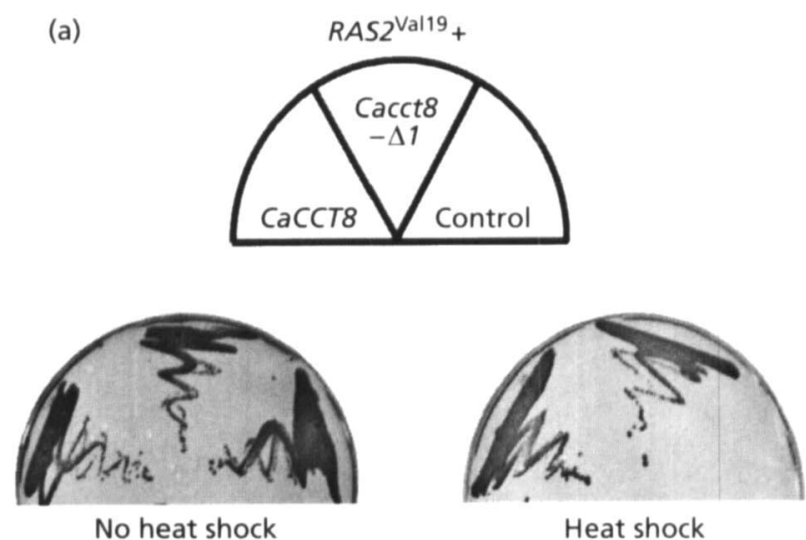

Heat shock

(b)

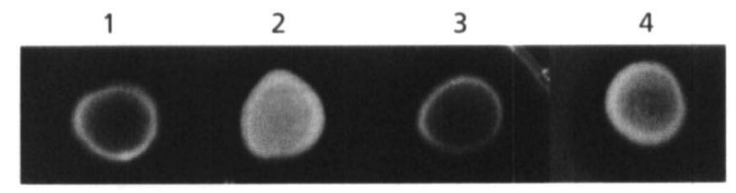

Fig. 2. Suppression of RAS2 $2^{\text {Val19 }}$ phenotypes by chaperonin subunits. (a) Suppression of heat sensitivity. $S$. cerevisiae strain B76/4 was transformed by YCplac22RAS2 valig and with a second plasmid containing CaCCT8 (YEplacGal1CCT8), Cacct8- $\Delta 1$ (YEplac195Gal10-RFG1) or no CCT gene (Control; YEplacGal1/10). Transformants were grown in SGal medium to stationary phase, heat-shocked at $50^{\circ} \mathrm{C}$ for $30 \mathrm{~min}$ (or not heatshocked) and plated on SD. (b) Restoration of glycogen accumulation. Strains were grown in liquid YPGal, spotted on solid YPGal and grown at $30^{\circ} \mathrm{C}$ for $1 \mathrm{~d}$. The accumulation of glycogen in colonies was visualized by iodine staining. (1) Glycogen accumulation (dark coloration) of a control transformant not expressing RAS2 ${ }^{\text {Val19 }}$ (YEplacGal1/10; YCplac22); (2) lack of glycogen accumulation in a transformant carrying RAS2 ${ }^{\text {Val19 }}$ (YEplacGal1/10; YCplac22RAS2 ${ }^{\text {Val19). }}$; Restoration of glycogen accumulation occurred in RAS2 $2^{\text {Valig }}$ transformants (YCplac22RAS2 ${ }^{\text {Val19) }}$ ) expressing (3) Cacct8- $\Delta 1$ (YEplac195Gal10-RFG1) or (4) CaCCT8 (YEplacGal1CCT8).

To examine chaperonin effects on sporulation we used the diploid strain VW1D as the host for the RAS2 $2^{\text {val19. }}$ encoding plasmid (YCplac22RAS2 ${ }^{\text {Val19 }}$ ), because strain B76/4 sporulates poorly. In this experiment we used a plasmid containing the Cacct8- $\Delta 1$ allele under control of the galactose-inducible GAL10 promoter (YEplac195Gal10-RFG1). RAS2 ${ }^{\text {Val19 }}$-expressing control strains (carrying plasmids YCplac22RAS2 ${ }^{\text {Val19 }}$ and YEplac195) did not sporulate, as expected, in either glucose- or galactose-containing sporulation media $(0 \%$ asci after 5-7 d). On the other hand, a transformant containing YCplac22RAS2 $^{\text {Val19 }}$ and YEplac195Gal10-RFG1 sporulated significantly only if the expression of Cacct8- $\Delta 1$ was induced by pregrowth in galactose $(17 \%$ asci); after pregrowth on glucose medium sporulation was reduced due to the low levels of Cacct $8-\Delta 1$ expression ( $3 \%$ asci).

We tested the accumulation of storage carbohydrates in a $R A S 2^{\text {Val19 }}$-carrying strain using iodine staining of 
(a)

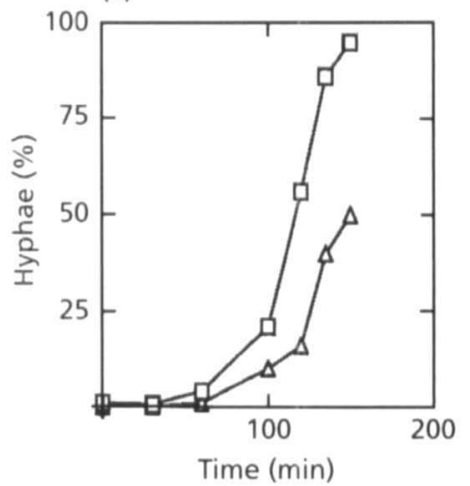

(b)

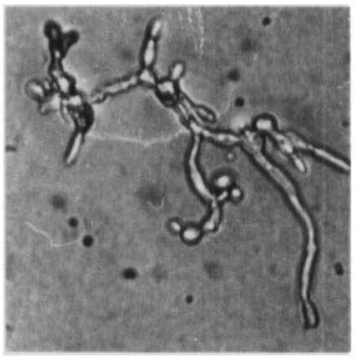

(i)

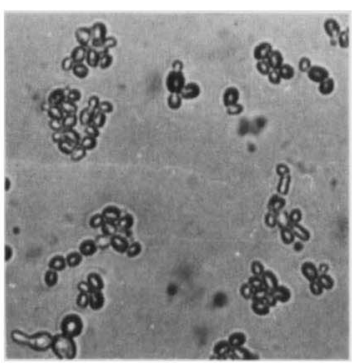

(ii)

(c)

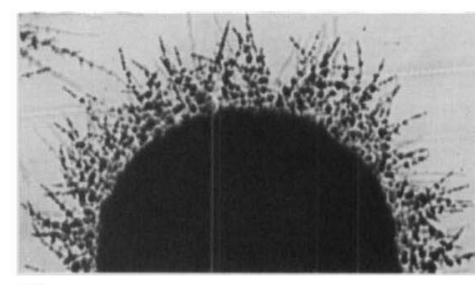

(i)

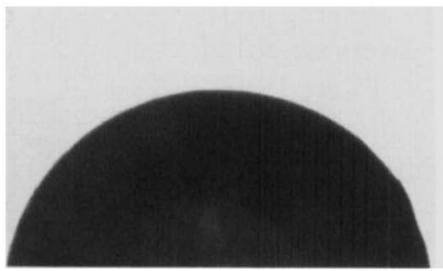

(ii)

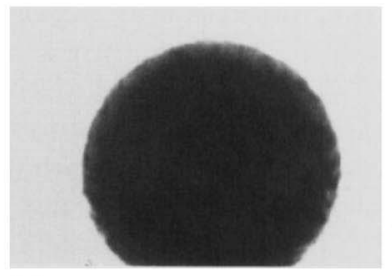

(iii)

Fig. 3. Chaperonins suppress hyphal morphogenesis of $C$. albicans. Strain CAI4 was transformed with plasmid pBI1-CCT8 expressing CaCCT8 or with a control plasmid (pBI-1). Hyphae were induced by (a) $\mathrm{N}$-acetylglucosamine in liquid medium, (b) on agar containing $1 \%$ serum or (c) on Spider plates. (a, $\square),(b, i)$ and $(c, i)$ are the results of the control strain $\mathrm{CAI} 4(\mathrm{pBI}-1) ;(\mathrm{a}, \triangle),(\mathrm{b}, \mathrm{ii})$ and $(\mathrm{c}, \mathrm{ii})$ are the results of transformant CAI4(pBI1-CCT8). The cellular phenotype on serum plates (b) is shown after $3 \mathrm{~h}$ growth; the colony phenotype on Spider plates (c) was determined after $5 \mathrm{~d}$ growth at $37^{\circ} \mathrm{C}$. The phenotype of the heterozygous strain FR1 (CaCCT8/Cacct8) on Spider plates is shown in (c, iii).

colonies (Fig. 2b). Dark coloration of colonies, indicating glycogen accumulation within cells, was observed for a control strain but not for a transformant expressing RAS2 ${ }^{\text {Val19 }}$. The simultaneous expression of CaCCT8 or Cacct8- $\Delta 1$ was able to restore glycogen accumulation to wild-type levels, even in the presence of $R A S 2^{\text {val19 }}$.

The suppression of all RAS2 ${ }^{\text {Val19 }}$ phenotypes, related or unrelated to morphogenesis, suggested that the $C$. albicans chaperonin subunits produced in the heterologous host act as dominant-negative inhibitors of a component of the Ras2 signal transduction pathway.

\section{CaCCT8 suppresses hyphal development in C. albicans}

True septated hyphae in C. albicans arise because of at least two signalling pathways operative either on certain solid media (Köhler \& Fink, 1996; Liu et al., 1994) or in liquid media in the presence of certain positive factors, including serum or GlcNAc (Lo et al., 1997; Stoldt et al., 1997). To test if any hyphal induction pathway is affected by $\mathrm{CaCCT} 8$ overexpression, we constructed pBI1-CCT8, in which CaCCT8 is under transcriptional control of the glucose-regulated PCK1 promoter (Leuker et al., 1997). Transformants of C. albicans strain CAI4 carrying either pBI1-CCT8 or the 'empty' control vector
pBI-1 were tested for hyphal morphogenesis after pregrowth in $1 \%$ Casamino acids (promoter induction) or SD medium (promoter repression).

Transformants carrying pBI-1 did not show differences in growth rates compared to the control strain in any medium and irrespective of the induction of the PCK1 promoter. However, pBI1-CCT8 transformants grown in Casamino acids and shifted to liquid medium containing $1 \mathrm{mM} \mathrm{GlcNAc}$ showed a reproducible delay in the formation of hyphae compared to control transformants carrying pBI-1; a typical result obtained with several independent transformants is shown in Fig. $3(a)$. Under these conditions hyphal morphogenesis was not abolished completely and after about $4 \mathrm{~h}$ induction both the pBI1-CCT8 and pBI-1 transformants had formed hyphae equally. No differences in the rate of hyphal formation were observed if the transformants were pregrown in SD medium, when the PCK1 promoter is repressed, suggesting that $\mathrm{CaCCT} 8$ overexpression is the cause for the delay of morphogenesis. A suppression of hyphal growth, but not a complete block, by $\mathrm{CaCCT} 8$ overexpression was also observed on solid plates containing $1 \%$ serum (Fig. $3 \mathrm{~b}$ ). Transformants were also tested on Spider medium (Liu et al., 1994) for hyphal induction. After $5 \mathrm{~d}$ growth, control cells had formed visible lateral hyphae emerging from colonies (Fig. 3c, i), 

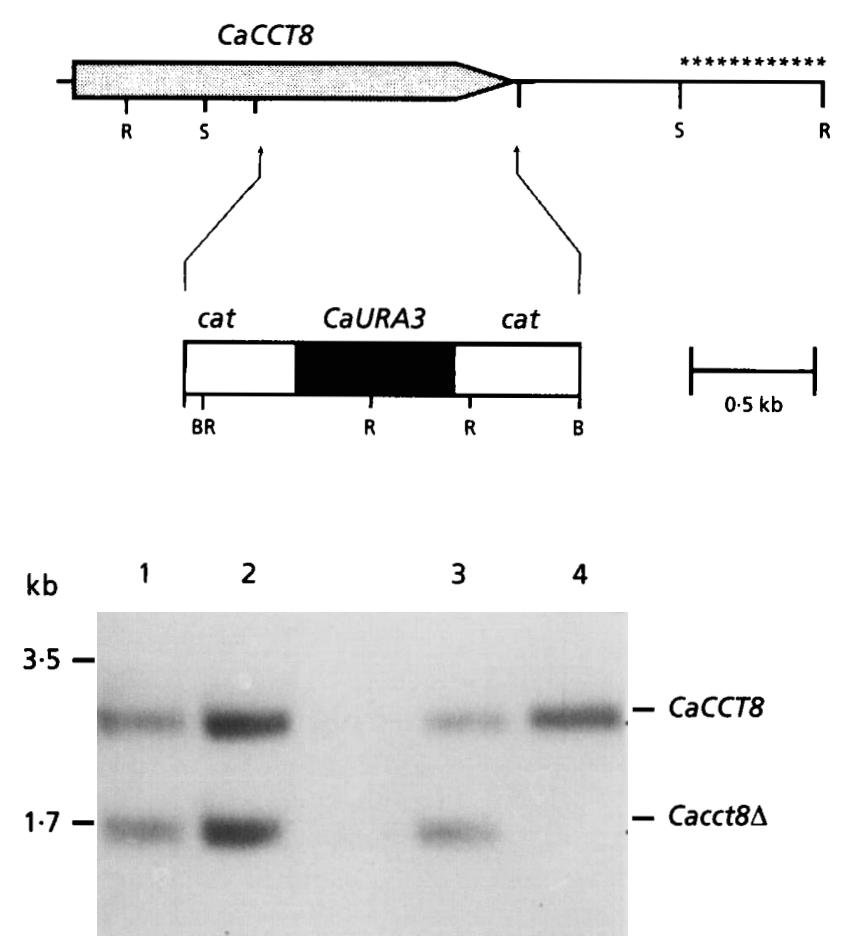

Fig. 4. Disruption of CCT8 alleles. The genomic fragment carrying CaCCT8 (grey arrow) is shown on top, along with the insertion site of the cat-URA3-cat cassette. B, BamHI; R, EcoRI; $\mathrm{S}$, Sall. The $4.5 \mathrm{~kb}$ Sall disruption fragment was transformed into strain CAI4. DNA of transformants (lanes 1-3) and of CAl4 (lane 4) was analysed after cutting with EcoRI and Southern blotting using a CaCCT8 probe fragment (asterisks). Transformants FR1$1,2,3$ (lanes 1-3, respectively) contain one intact allele (CaCCT8; $2.8 \mathrm{~kb})$ and one disrupted allele (Cacct8 $; 1.9 \mathrm{~kb})$.

while colonies formed by CAI4(pBI1-CCT8) cells did not show any hyphae (Fig. 3c, ii). Overexpression of the Cacct8- $\Delta 1$ allele in C. albicans under transcriptional control of the constitutive ACT1 promoter (pCARSAct-RFG1) led to an identical partial suppression of hyphal growth compared to the PCK1p-CaCCT8 transformants described above. Furthermore, transformants carrying pCARS-Act-RFG1 showed a slowed hyphal formation in liquid medium containing $1 \%$ GlcNAc and on solid plates containing $1 \%$ serum (data not shown).

These results demonstrate that $\mathrm{CaCCT} 8$ overexpression partially suppresses hyphal formation induced by the serum/GlcNAc pathway and completely blocks morphogenesis induced on Spider medium in C. albicans.

\section{Lowered CaCCT8 gene dosage in a heterozygous strain blocks $C$. albicans morphogenesis}

The above results suggested that excess levels of chaperonin subunits can act as dominant-negative suppressors of morphogenesis and Ras phenotypes by inhibiting a component of the Ras $2 p$ signalling pathway. As a mechanism for this effect we hypothesized that overproduced chaperonins are not assembled into a functional Cct complex, which presumably requires all individual subunits Cct $1 \mathrm{p}-\mathrm{Cct} 8 \mathrm{p}$. Instead, orphan subunits would still be able to bind to their folding target proteins, leading to a sequestration of unfolded target proteins and, therefore, a lowered level of active target protein within cells. Conversely, lowering chaperonin levels within cells would also be predicted to reduce the activity of the target protein. To test if morphogenesis is blocked at reduced chaperonin levels, as in the case of chaperonin overproduction, we attempted to disrupt the C. albicans $\mathrm{CaCCT8}$ gene.

Using the 'URA3 blaster' method (Fonzi \& Irwin, 1993) we were able to disrupt one CaCCT8 allele in strain CAI4 without difficulty by using a URA3-containing disruption fragment. In Southern blots of genomic DNA a $2.8 \mathrm{~kb}$ EcoRI fragment, representing the wild-type allele, and a $1.9 \mathrm{~kb}$ fragment, corresponding to the disrupted allele, were found in three independent mutants (Fig. 4). Next we isolated $\mathrm{Ura}^{-}$mutant derivatives of the disruptant strains, which had arisen because of recombination between the cat repeats flanking the $U R A 3$ selection marker; $\mathrm{Ura}^{-}$derivatives were used in a second round of transformation with the disruption fragment in an attempt to mutate the second CaCCT8 allele. Among 54 transformants no double-deleted mutant could be obtained (as determined by Southern blotting), suggesting that the deletion of both CaCCT8 alleles in C. albicans is lethal, as has been observed for $S$. cerevisiae mutants lacking individual Cct subunits (reviewed by Stoldt et al., 1996). Standard protocols were used to test the ability of the CaCCT8/Cacct8 heterozygous strain to form filaments. Remarkably, a clear phenotypic difference of the heterozygous disruptant compared to the parental strain was observed on solid Spider medium. Under these conditions only the parental strain, but not the $\mathrm{CaCCT8/Cacct8}$ heterozygous strain, was able to form hyphae (Fig. 3c, iii). On the other hand, hyphae were formed at wild-type rates in the presence of $5 \%$ serum in liquid or on solid media.

These results suggest that both excess and lowered levels of chaperonins can abolish morphogenesis of C. albicans, which may occur in both cases because of lowered activity of a protein in the Ras 2 pathway.

\section{DISCUSSION}

In a search for suppressors of C. albicans morphogenesis we discovered that high levels of an individual subunit of the eukaryotic Cct chaperonin complex lead to a specific block of fungal morphogenesis. We found that overexpression of CaCCT8, encoding subunit 8 of the Cct complex, inhibits hyphal morphogenesis in $C$. albicans and that its expression in the heterologous host $S$. cerevisiae blocks pseudohyphal growth. No effects of CaCCT8 overexpression on growth and on cytological appearance of the yeast growth forms of both fungi were observed. The analyses revealed that, rather than morphogenesis per se, it is very likely that a component of the Ras signal transduction pathway is inhibited by extraneous $\mathrm{CaCct} 8 \mathrm{p}$ levels. These results suggest a yet undefined component of fungal morphogenesis path- 
ways, as well as a novel cytosolic folding target protein of the Cct complex.

\section{A component of the Ras pathway as the target of suppression by CaCct8p}

Recent evidence indicates that Ras $2 p$ activates two signal transduction pathways as a response of $S$. cerevisiae diploids to nitrogen starvation. One pathway, whose activity can be monitored by the $F G(T y A)$ promoter (Mösch et al., 1996) leads to the activation of a conserved MAP kinase (Kss1p) and phosphorylation of the transcription factor Ste12p (Liu et al., 1993; Madhani \& Fink, 1997; Madhani et al., 1997). The second pathway includes cAMP, whose levels are upregulated by both Ras $2 p$ and Gpa2p, a G-protein $\alpha$ subunit (Kübler et al., 1997; Lorenz \& Heitman, 1997). It is still not clear, if cAMP exerts its effects via PKA (TPK1-3 gene products; Toda et al., 1987), or is independent of PKA. A function of PKA in yeast morphogenesis can be deduced from the suppression pattern of the heterologous $\mathrm{CaCct} 8 \mathrm{p}$ chaperonin subunit in $S$. cerevisiae described here. CaCct8p did not block pseudohyphal growth induced by Cdc42p or Cph1p (Ste12p), indicating that the 'chaperonin effect' is not due to a block of the 'MAP kinase branch' of signal transduction pathways leading to morphogenesis. This result could be confirmed by measurements of the $F G(T y A)$ promoter, which was unaffected by $C a C C T 8$ expression. Nevertheless, morphogenesis stimulated by $R A S 2^{\text {val19 }}$ could be effectively suppressed, suggesting that the target of suppression is located in the "cAMP branch' of the morphogenetic signalling pathways. Either PKA itself or a functionally related protein could be the target of suppression, because all additional known RAS2 $2^{\text {Val19 }}$ phenotypes mediated by PKA are inhibited: heat sensitivity, lack of sporulation and lack of glycogen/trehalose accumulation phenotypes (Durnez et al., 1994; Ruis \& Schüller, 1995; Toda et al., 1985) were all restored to wild-type phenotypes by simultaneous expression of $\mathrm{CaCCT8}$. It has been reported that overexpression of TPK1, encoding PKA, did not stimulate filamentation in a $\Sigma 1298 \mathrm{~b}$ derivative (Mösch et al., 1996), while we found this to be the case in strains of different genetic backgrounds ( $F$. Rademacher \& J. F. Ernst, unpublished results). Thus, it is possible that specific PKA isoforms (possibly to different extents depending on the genetic background of strains) are required for morphogenesis via the Ras2/cAMP pathway.

Morphogenetic pathways in C. albicans are less defined than in S. cerevisiae. Mutations in the CST20,HST7 and CPH1 genes (homologues of STE20, STE7 and STE12 genes of $S$. cerevisiae, respectively) block hyphal development on solid Spider medium, but have no effect on hyphal growth induced by positive signals, such as serum components (Köhler \& Fink, 1996; Leberer et al., 1996; Liu et al., 1993). Most likely, the 'starvation pathway' on Spider medium seems to represent the functional equivalent of the $S$. cerevisiae MAP kinase pathway. The observed suppression of both the star- vation and the positive signals pathway by $\mathrm{CaCct} 8 \mathrm{p}$ suggests that one or more common elements occur in both pathways. In C. albicans, exogenous cAMP stimulates hyphal growth, indicating a role for cAMP and suggesting a role for PKA in hyphal morphogenesis (Niimi et al., 1980; Niimi, 1996). Stimulation of fungal morphogenesis via cAMP and PKA has also been reported for Ustilago maydis (Gold et al., 1994) and other fungi (Kronstad, 1997).

Our finding that morphogenesis induced by PHD1 or EFG1 can be effectively suppressed by CaCCT 8 expression suggests that their gene products are components of the cAMP morphogenetic pathway. Phd1p contains a region that is highly homologous to the Sok2 protein, which appears to act as a suppressor of pseudohyphal growth (Gimeno \& Fink, 1994; Ward et al., 1996) and to bHLH proteins regulating morphogenesis in other fungal species, including Efg1p of $C$. albicans and StuA of Aspergillus nidulans (Miller et al., 1992; Stoldt et al., 1997). Genetic evidence suggests that the homologous Sok2 protein acts downstream of PKA to regulate the expression of genes important in growth and development (Ward et al., 1996). Possibly, Phd1p and Efg1p may assume similar functions in the cAMP pathway, conceivably as direct or indirect phosphorylation targets of PKA.

\section{Mechanism of morphogenesis suppression by CaCct8p}

It has been established that the Cct complex has a narrow substrate specificity and mainly folds newly synthesized actin and tubulin, but also binds to small amounts of unidentified proteins in vivo (Sternlicht $e t$ al., 1993; Ursic et al., 1994; Yaffe et al., 1992). Our results suggest that a protein of the Ras2/cAMP signalling pathways is one of the unidentified folding targets of the Cct complex. We propose that excess $\mathrm{CaCct} 8$ proteins within cells that cannot be assembled into a functional Cct complex [which appears to consist of equimolar amounts of eight specific subunits (Lin $e t$ al., 1997)] still bind to their target proteins, but are unable to fold them. Sequestration of target proteins (e.g. PKA) may decrease their cellular activity levels and thereby lead to a dominant-negative phenotype (e.g. prevention of hyphal morphogenesis). Conceivably, chaperonin target proteins existing at low levels within cells are affected more significantly in their cellular activity levels than abundant target proteins, such as actin or tubulin, whose inhibition could cause a general growth defect. We do not consider it likely that our results are due to a positive function of excess chaperonins, as has been suggested from in vitro experiments (Zahn et al., 1996), because even a deleted chaperonin (CaCct8- $\Delta 1 \mathrm{p})$ lacking the ATP-binding region that is required for protein folding (Kubota et al., 1995) was as active a suppressor as the full-length chaperonin. Possibly, the 'antimorphic' nature of some alleles of the mouse $T$ locus is due to dominant-negative effects of full-length or deleted versions of chaperonin Tcp$1 / \mathrm{Cct} 1$, which is encoded by this locus (MacMurray $\&$ 
Shin, 1988). Recently, overexpression of ScCCT6 was reported to restore growth and polarized distribution of the actin cytoskeleton in a tor 2 mutant (Schmidt et al., 1996). This result may be due to a dominant-negative effect, e.g. on Sac7p, since $s a c 7$ mutations restore the growth of tor2 strains (Schmidt et al., 1997).

\section{Both CaCCT8 alleles are required for $\mathrm{C}$. albicans morphogenesis}

A dominant-negative function of excess chaperonins in morphogenesis implies that the respective target protein is an essential component of the suppressed pathway. We predicted therefore, that a reduced dosage of a single chaperonin subunit would diminish the concentration of functional Cct complexes and therefore abolish or delay hyphal morphogenesis. Although we were not able to delete both $C a C C T 8$ genes in C. albicans, in agreement with the lethality of single $c c t$ mutants of $S$. cerevisiae (reviewed by Stoldt et al., 1996), we found that in a $\mathrm{CaCCT8/Cacct8}$ heterozygous strain hyphae no longer could be induced on Spider medium. Hyphal induction by serum was still possible, suggesting that the 'starvation pathway' is more affected by reduced activity of the target protein than the 'serum pathway', in agreement with our finding that excess chaperonins completely abolish morphogenesis on Spider medium, while only partially affecting the response to serum.

In summary, our findings (1) that overproduction and lowering of $\mathrm{CaCct} 8 \mathrm{p}$ levels lead to comparable phenotypes, (2) that pseudohyphal morphogenesis induced by high activity of the Ras pathway, but not the MAP kinase pathway, is suppressed by CaCct8p and (3) that morphogenesis-unrelated phenotypes of Ras $2^{\mathrm{Val19}}$ are suppressed by $\mathrm{CaCct8p}$ strongly support our hypothesis that excess chaperonins inhibit a specific target protein in the Ras pathway.

\section{ACKNOWLEDGEMENTS}

We thank A. Datta, E. Dubois, G. Fink, D. Johnson, P. Kötter, P. Magee, H.-U. Mösch, B. Stevenson and M. Wigler for strains or plasmids. Supported by grant BMH4-CT96-0310 of the European Community and by the Deutsche Forschungsgemeinschaft. We thank H.-U. Mösch and M. Brandriss for helpful comments.

\section{REFERENCES}

Brandriss, M. C. (1983). Proline utilization in Saccharomyces cerevisiae: analysis of the cloned PUT2 gene. Mol Cell Biol 3, $1846-1856$

Cannon, R. D., Jenkinson, H. F. \& Shepherd, M. G. (1992). Cloning and expression of Candida albicans $A D E 2$ and proteinase genes on a replicative plasmid in C. albicans and in Saccharomyces cerevisiae. Mol Gen Genet 235, 453-457.

Christianson, T. W., Sikorski, R. S., Dante, M., Shero, J. H. \& Hieter, P. (1992). Multifunctional yeast high-copy-number shuttle vectors. Gene 110, 119-122.

Delbruck, S. \& Ernst, J. F. (1993). Morphogenesis-independent regulation of actin transcript levels in the pathogenic yeast Candida albicans. Mol Microbiol 10, 859-866.
Durnez, P., Pernambuco, M. B., Oris, E., Arguelles, J.-C., Mergelsberg, H. \& Thevelein, J. M. (1994). Activation of trehalase during growth induction by nitrogen sources in the yeast Saccharomyces cerevisiae depends on the free catalytic subunits of cAMPdependent protein kinase, but not on functional Ras proteins. Yeast 10, 1049-1064.

Fonzi, W. A. \& Irwin, M. Y. (1993). Isogenic strain construction and gene mapping in Candida albicans. Genetics 134, 717-728.

Gavrias, V., Andrianopoulos, A., Gimeno, C. J. \& Timberlake, W. E. (1996). Saccharomyces cerevisiae TEC1 is required for pseudohyphal growth. Mol Microbiol 19, 1255-1263.

Gietz, R. D. \& Sugino, A. (1988). New yeast-Escherichia coli shuttle vectors constructed with in vitro mutagenized yeast genes lacking six-base pair restriction sites. Gene 74, 527-534.

Gimeno, C. J. \& Fink, G. R. (1994). Induction of pseudohyphal growth by overexpression of PHD1, a Saccharomyces cerevisiae gene related to transcriptional regulators of fungal development. Mol Cell Biol 14, 2100-2112.

Gimeno, C. J., Ljungdahl, P. O., Styles, C. A. \& Fink, G. R. (1992). Unipolar cell divisions in the yeast $S$. cerevisiae lead to filamentous growth: regulation by starvation and RAS. Cell 68, 1077-1090.

Gold, S., Duncan, G., Barrett, K. \& Kronstad, J. (1994). cAMP regulates morphogenesis in the fungal pathogen Ustilago maydis. Genes Dev 8, 2805-2816.

Johnston, M. \& Davis, R. W. (1984). Sequences that regulate the divergent GAL1-GAL10 promoter in Saccharomyces cerevisiae. Mol Cell Biol 4, 1440-1448.

Kelly, R., Miller, A. M. \& Kurtz, M. B. (1988). One-step gene disruption by cotransformation to isolate double auxotrophs in Candida albicans. Mol Gen Genet 214, 24-31.

Köhler, J. R. \& Fink, G. R. (1996). Candida albicans strains heterozygous and homozygous in mitogen-activated protein kinase signalling components have defects in hyphal development. Proc Natl Acad Sci USA 93, 13223-13228.

Kron, S. J. \& Gow, N. A. R. (1995). Budding yeast morphogenesis: signalling, cytoskeleton and cell cycle. Curr Opin Cell Biol 7, 845-855.

Kronstad, J. W. (1997). Virulence and cAMP in smuts, blasts and blights. Trends Plant Sci 2, 193-199.

Kubler, E., Mösch, H.-U., Rupp, S. \& Lisanti, M. P. (1997). Gpa2p, a G-protein $\alpha$-subunit, regulates growth and pseudohyphal development in Saccharomyces cerevisiae via a cAMP-dependent mechanism. J Biol Chem 272, 20321-20323.

Kubota, H., Hynes, G. \& Willison, K. (1995). The chaperonin containing $t$-complex polypeptide 1 (TCP-1). Multisubunit machinery assisting in protein folding and assembly in the eukaryotic cytosol. Eur J Biochem 239, 3-16.

Laloux, I., Jacobs, E. \& Dubois, E. (1994). Involvement of SRE element of Ty1 transposon in TEC1-dependent transcriptional activation. Nucleic Acids Res 22, 999-1005.

Leberer, E., Harcus, D., Broadbent, I. D., Clark, K. L., Dignard, D., Ziegelbauer, K., Schmidt, A., Gow, N. A. R., Brown, A. J. P. \& Thomas, D. Y. (1996). Signal transduction through homologs of the Ste20p and Ste7p protein kinases can trigger hyphal formation in the pathogenic fungus Candida albicans. Proc Natl Acad Sci USA 93, 13217-13222.

Leuker, C. E. \& Ernst, J. F. (1994). Toxicity of a heterologous leucyl-tRNA (anticodon CAG) in the pathogen Candida albicans: in vivo evidence for non-standard decoding of CUG codons. Mol Gen Genet 245, 212-217.

Leuker, C. E., Sonneborn, A., Delbrück, S. \& Ernst, J. F. (1997). Sequence and promoter regulation of the PCK1 gene encoding 
phosphoenolpyruvate carboxykinase of the fungal pathogen Candida albicans. Gene 192, 235-240.

Lin, P., Cardillo, T. S., Richard, L. M., Segal, G. B. \& Sherman, F. (1997). Analysis of mutationally altered forms of the Cct6 subunit of the chaperonin from Saccharomyces cerevisiae. Genetics 147, 1609-1633.

Liu, H., Styles, C. A. \& Fink, G. R. (1993). Elements of the yeast pheromone response pathway required for filamentous growth of diploids. Science 262, 1741-1744.

Liu, H., Kohler, J. \& Fink, G. R. (1994). Suppression of hyphal formation in Candida albicans by mutation of a STE12 homolog. Science 266, 1723-1725.

Lo, H.-J., Köhler, J. R., Didomenico, B., Loebenberg, D., Cacciapuoti, A. \& Fink, G. R. (1997). Nonfilamentous C. albicans mutants are avirulent. Cell 90, 939-949.

Lorenz, M. C. \& Heitman, J. (1997). Yeast pseudohyphal growth is regulated by GPA2, a $\mathrm{G}$ protein $\alpha$ homolog. EMBO $J \mathbf{1 6}$, 7008-7018.

Losberger, C. \& Ernst, J. F. (1989). Sequence and transcript analysis of the C. albicans URA3 gene encoding orotidine- 5 '-phosphate decarboxylase. Curr Genet 16, 153-157.

MacMurray, A. \& Shin, H.-S. (1988). The antimorphic nature of the $T c$ allele at the mouse $T$ locus. Genetics 120, 545-550.

Madhani, H. D. \& Fink, G. R. (1997). Combinatorial control required for the specificity of yeast MAPK signaling. Science $\mathbf{2 7 5}$, 1314-1317.

Madhani, H. D., Styles, C. A. \& Fink, G. R. (1997). MAP kinases with distinct inhibitory functions impart signaling specificity during yeast differentiation. Cell 91, 673-684.

Miller, K. Y., Wu, J. \& Miller, B. L. (1992). StuA is required for cell pattern formation in Aspergillus. Genes Dev 6, 1770-1782.

Mosch, H.-U., Roberts, R. \& Fink, G. R. (1996). Ras2 signals via the $\mathrm{Cdc} 42 / \mathrm{Ste} 20 /$ mitogen-activated kinase module to induce filamentous growth in Saccharomyces cerevisiae. Proc Natl Acad Sci USA 93, 5352-5356.

Niimi, M. (1996). Dibutyryl cyclic AMP-enhanced germ tube formation in exponentially growing Candida albicans cells. Fungal Genet Biol 20, 79-83.

Niimi, M., Niimi, K., Tokunaga, J. \& Nakayama, H. (1980). Changes in cyclic nucleotide levels and dimorphic transition in Candida albicans. J Bacteriol 142, 1010-1014.

Odds, F. C. (1988). Candida and Candidosis. London: Baillière Tindall.

Ruis, H. \& Schüller, C. (1995). Stress signaling in yeast. BioEssays 17, 959-965.

Schmidt, A., Kunz, J. \& Hall, M. N. (1996). TOR2 is required for organization of the actin cytoskeleton in yeast. Proc Natl Acad Sci USA 93, 13780-13785.

Schmidt, A., Bickle, M., Beck, T. \& Hall, M. N. (1997). The yeast phosphatidylinositol kinase homolog TOR2 activates RHO1 and RHO2 via the exchange factor ROM2. Cell 88, 531-542.

Sherman, F., Fink, G. \& Hicks, J. (1986). Methods in Yeast Genetics. Cold Spring Harbor, NY: Cold Spring Harbor Laboratory.

Singh, P., Ganesan, K., Malathi, K., Gosh, D. \& Datta, A. (1994). $A C P R$, a STE12 homologue from Candida albicans, is a strong inducer of pseudohyphae in Saccharomyces cerevisiae haploids and diploids. Biochem Biophys Res Commun 205, 1079-1085.

Sternlicht, H., Farr, G. W., Sternlicht, M. L., Driscoll, J. K., Willison, K. \& Yaffe, M. B. (1993). The t-complex polypeptide 1 complex is a chaperonin for tubulin and actin in vivo. Proc Natl Acad Sci USA 90, 9422-9426.

Stoldt, V., Rademacher, F., Kehren, V., Ernst, J. F., Pearce, D. A. \& Sherman, F. (1996). The Cct eukaryotic chaperonin subunits of Saccharomyces cerevisiae and other yeasts. Yeast 12, 523-529.

Stoldt, V. R., Sonneborn, A., Leuker, C. \& Ernst, J. F. (1997). Efg1, an essential regulator of morphogenesis of the human pathogen Candida albicans, is a member of a conserved class of bHLH proteins regulating morphogenetic processes in fungi. EMBO J 16, 1982-1991.

Toda, T., Uno, I., Ishikawa, T., Powers, S., Kataoka, T., Broek, D., Cameron, S., Broach, J., Matsumoto, K. \& Wigler, M. (1985). In yeast, $R A S$ proteins are controlling elements of adenylate cyclase. Cell 40, 27-36.

Toda, T., Cameron, S., Sass, P., Zoller, M. \& Wigler, M. (1987). Three different genes in $S$. cerevisiae encode the catalytic subunits of the cAMP-dependent protein kinase. Cell 50, 277-287.

Ursic, D., Sedbrook, J. C., Himmel, K. L. \& Culbertson, M. E. (1994). The essential yeast $T c p 1$ protein affects actin and microtubules. Mol Biol Cell 5, 1065-1080.

Ward, M. P., Gimeno, C. J., Fink, G. R. \& Garrett, S. (1996). SOK2 may regulate cyclic AMP-dependent protein kinase-stimulated growth and pseudohyphal development by repressing transcription. Mol Cell Biol 15, 6854-6863.

Yaffe, M. B., Farr, G. W., Miklos, D., Horwich, A. L., Sternlicht, M. L. \& Sternlicht, H. (1992). TCP1 complex is a molecular chaperone in tubulin biogenesis. Nature 358, 245-248.

Zahn, R., Buckle, A. M., Perrett, S., Johnson, C. M., Corrales, F. J., Golbik, R. \& Fersht, A. R. (1996). Chaperone activity and structure of monomeric polypeptide binding domains of GroEL. Proc Natl Acad Sci USA 93, 15024-15029.

Ziman, M., O'Brien, J. M., Oullette, L. A., Church, W. R. \& Johnson, D.I. (1991). Mutational analysis of $C D C 42 S c$, a Saccharomyces cerevisiae gene that encodes a putative GTPbinding protein involved in the control of cell polarity. Mol Cell Biol 11, 3537-3544.

Received 13 May 1998; revised 6 August 1998; accepted 14 August 1998 